\title{
INVESTIGACIÓN
}

\section{Formación de compuestos fluorescentes durante el almacenamiento refrigerado de sardina (Sardina pilchardus): comparación con índices de alteración lipídica}

\author{
Por S. P. Aubourg y R. Pérez-Martín
}

Instituto de Investigaciones Marinas (CSIC), c/ Eduardo Cabello, 6, 36208-Vigo. Fax (986) 292762

\section{RESUMEN}

Formación de compuestos fluorescentes durante el almacenamiento refrigerado de sardina (Sardina pilchardus): comparación con índices de alteración lipídica

Durante 16 días se almacenaron sardinas en hielo. A lo largo de este tiempo se estudió la formación de compuestos fluorescentes a distintos máximos de longitud de onda de excitación/emisión con el fin de seguir las posibles variaciones de estos compuestos producidas al aumentar el tiempo de almacenamiento; las medidas de fluorescencia se realizaron sobre las fases acuosa y orgánica (lipídica) resultantes de la extracción de lípidos. Asimismo, estos análisis se compararon con medidas tradicionales de hidrólisis (contenido en ácidos grasos libres, AGL) y oxidación (contenido en dienos conjugados, índice de ácido tiobarbitúrico, TBA) lipídicas.

Los índices clásicos de alteración lipídica no proporcionaron una buena medida del estado de alteración en el caso de encontrarse ésta en estado avanzado. Así, el contenido en dienos reflejó un valor creciente hasta el noveno día, produciéndose después un descenso paulatino. Por su parte, las medidas de AGL e índice de TBA mostraron su valor máximo el decimotercer día de almacenamiento, produciéndose posteriormente un ligero descenso.

Se observó que a medida que aumentaba el tiempo de almacenamiento se producía un incremento en la fluorescencia de la mayoría de los máximos considerados ( $393 / 463 \mathrm{~nm}, 446 / 490 \mathrm{~nm}$ y $479 / 516 \mathrm{~nm}$ ), mientras que otros tenían tendencia a disminuir $(327 / 415 \mathrm{~nm}$, especialmente); como resultado global se producía un incremento relativo de la fluorescencia de los máximos de mayor longitud de onda frente a los de menor longitud de onda.

La relación entre dos de los máximos de fluorescencia estudiados (393/463 y 327/415) experimentó un incremento progresivo respecto al tiempo de almacenamiento de la muestra de sardina; el estudio de esta relación en la fase acuosa reflejó una mejor correlación $(r=0,8533)$ que en el caso de la fase orgánica $(r=0,7457)$. Con los resultados del presente trabajo se concluye que la medida de compuestos de interacción a través de sus propiedades fluorescentes resulta útil como medida de la alteración durante el almacenamiento en estado refrigerado.

PALABRAS-CLAVE: Almacenamiento - Calidad Compuesto fluorescente - Hidrólisis lipídica - Oxidación lipídica - Refrigeración - Sardina.

\section{SUMMARY}

Fluorescent compounds formation during sardine (Sardina pilchardus) chilling: Comparison with lipid damage indexes.
Sardine was stored at $0^{\circ} \mathrm{C}$ on ice during 16 days. During this time the fluorescent compounds formation was studied at different wavelength maxima in order to assert quality changes; fluorescence analyses were undergone on the aqueous and organic (lipids) phases resulting from the lipid extraction. Quality measures of lipid hydrolysis (free fatty acids content, FFA) and oxidation (conjugated diene formation and thiobarbituric acid, TBA, test) were also carried out.

Traditional indexes of lipid damage did not assert deterioration in the case of advanced damage. Diene content reflected and increasing value till the day 9 of storage, and then a continuous decrease. Further, FFA content and TBA test showed the highest values on the day 13 of storage, followed by a slight decrease.

An increase in fluorescence content was observed in most of the excitation/emission maxima studied $(393 / 463 \mathrm{~nm}$, $446 / 490 \mathrm{~nm}$ and $479 / 516 \mathrm{~nm}$ ), at the time that others decreased $(327 / 415 \mathrm{~nm}$, specially); as an overall result, a relative increase in the maxima of higher wavelength was observed in comparison with the lower ones.

The ratio between two of the fluorescence maxima analyzed $(393 / 463 \mathrm{~nm}$ and $327 / 415 \mathrm{~nm})$ exhibited an overall increase respect to the time of chilled storage. The study of this ratio in the aqueous extract showed a better correlation $(r=0,8533)$ than in the case of the organic one $(r=0,7457)$. From the present results, it is concluded that the interaction compounds determination by mean of their fluorescent properties is useful as a damage measure during the chilled storage.

KEY-WORDS: Fluorescent compound - Lipid hydrolysis Lipid oxidation - Quality - Refrigeration - Sardine - Storage.

\section{INTRODUCCIÓN}

La calidad de los productos marinos tiene una estrecha relación con el contenido lipídico y su composición. El alto nivel en ácidos altamente insaturados (Ackman, 1989) puede llevar a cambios indeseables causados por el procesamiento y/o almacenamiento, con especial incidencia sobre la calidad del producto final (Cheftel y Cheftel, 1976; Pearson et al., 1977).

Se ha utilizado una gran diversidad de métodos al objeto de medir los productos de oxidación primaria (peróxidos) y secundaria (compuestos carbonílicos) formados durante la degradación lipídica en los alimentos (Melton, 1983; Kim y Labella, 1987). Sin 
embargo, se ha comprobado que ambos tipos de compuestos pueden reaccionar con constituyentes de los alimentos portadores de grupos amino (proteínas, péptidos pequeños, aminoácidos libres y fosfolípidos aminados) para producir compuestos de interacción con propiedades fluorescentes, aromáticas y portadores de coloración (Pokorny, 1977; Gardner, 1979; Zamora et al., 1986; Hidalgo et al., 1990).

La medida de estos compuestos de interacción a través de sus propiedades fluorescentes se usa con frecuencia como medida complementaria al objeto de estudiar el estado de calidad de un alimento (Maruf et al., 1990; Lubis y Buckle, 1990; Hasegawa et al., 1992). En el caso de los productos marinos, la formación de estos compuestos durante el procesamiento y/o almacenamiento se acelera debido a su alto contenido en ácidos poliinsaturados.

Estudios previos llevados a cabo en nuestro equipo han medido las propiedades fluorescentes a distintos máximos de longitud de onda de excitación/emisión de muestras de pescado sometidas a tratamientos térmicos (cocción y esterilización). Como resultado se observó un desplazamiento de la fluorescencia hacia máximos de longitud de onda mayores causado por el procesamiento y la presencia de productos de oxidación lipídica (Aubourg et al., 1992a, $1992 b$ y 1995a).

El objetivo del presente trabajo es estudiar la formación de compuestos fluorescentes durante el almacenamiento refrigerado de muestra de pescado. La medida de la fluorescencia se realiza a distintos máximos de excitación/emisión al objeto de estudiar el desplazamiento producido. Asimismo, la variación en propiedades fluorescentes se compara con otras medidas clásicas de alteración lipídica: hidrólisis (formación de ácidos grasos libres) y oxidación (índice de ácido tiobarbitúrico y formación de dienos conjugados).

\section{PARTE EXPERIMENTAL}

\subsection{Materia prima, almacenamiento y toma de muestra}

Se emplearon sardinas (Sardina pilchardus) capturadas (mes de junio) la noche anterior al inicio de la presente experiencia. Se mantuvieron directamente en hielo desde la captura hasta su llegada a nuestro Instituto. En ese momento, las sardinas sin eviscerar se distribuyeron en tres lotes distintos que se mantuvieron en hielo en una cámara termostatizada a $0^{\circ} \mathrm{C}$. Cada lote se estudió por separado en cada toma de muestra (0, 2, 6, 9, 13 y 16 días); se consideraron los resultados. obtenidos como media de las medidas de los tres lotes. Los análisis se realizaron sobre el músculo blanco proveniente de la mezcla de cuatro individuos de cada lote en cada muestreo.
La experiencia se prolongó hasta 16 días al objeto de tener una información completa de la variación de los distintos índices durante el almacenamiento refrigerado.

\subsection{Análisis básicos}

La determinación de humedad se realizó sobre muestras (1-2 g) homogeneizadas de músculo blanco que se mantuvieron durante 24 horas en una estufa a $105^{\circ} \mathrm{C}$ (Aursand et al., 1994). Los resultados se expresan como $\mathrm{g} \mathrm{H}_{2} \mathrm{O} / 100 \mathrm{~g}$ músculo.

Los lípidos se extrajeron de las muestras mediante el método de Bligh y Dyer (1959); su cuantificación se llevó a cabo según el método de Herber y Allen (1983). Los resultados se expresan como g lípidos/100 g músculo.

\subsection{Medidas de alteración lipídica}

El contenido en ácidos grasos libres (AGL) de los extractos lipídicos se determinó por el método de Lowry y Tinsley (1976) basado en la formación de un complejo con $(\mathrm{AcO})_{2}$ Cu-piridina. Los resultados se expresan como g AGL/100 g lípidos.

La formación de dienos conjugados se midió a 233 $\mathrm{nm}$ (Kim y Labella, 1987). El resultado se expresa de acuerdo a la siguiente fórmula (Smith et al., 1990):

$$
\text { Contenido en Dienos: } \frac{B \times V}{p}
$$

donde $\mathbf{B}$ es la absorbancia de la muestra utilizada en el ensayo, $\mathbf{p}$ es la cantidad de lípidos ( $\mathrm{mg}$ ) empleada y $V$ el volumen $(\mathrm{ml})$ en que estaban disueltos.

El índice de ácido tiobarbitúrico (TBA) (mg malonaldehído/Kg de muestra) se obtuvo a partir de un extracto de músculo en ácido tricloroacético al $5 \%$ en agua, de acuerdo con el método de Vyncke (1970).

\subsection{Medidas de fluorescencia}

Mediante el empleo de un espectrofotómetro de fluorescencia Perkin-Elmer LS 3B se analizaron los espectros de excitación y emisión de las fases acuosa y orgánica elaborada (extracto lipídico) resultantes de realizar la extracción lipídica mediante el método de Bligh y Dyer (1959).

Las medidas se realizaron en los siguientes máximos de excitación/emisión: $327 / 415 \mathrm{~nm}, 356 / 429 \mathrm{~nm}$, $393 / 463 \mathrm{~nm}, 446 / 490 \mathrm{~nm}, 479 / 516 \mathrm{~nm}$ y $528 / 556 \mathrm{~nm}$. Como estándar de fluorescencia se empleó una disolución de sulfato de quinina $\left(1 \mu \mathrm{g} / \mathrm{ml}\right.$ en $\left.\mathrm{SO}_{4} \mathrm{H}_{2} 0,05 \mathrm{M}\right)$. La fluorescencia de la muestra relativa a la del estándar se calculó usando la siguiente fórmula (Smith et al., 1990): 


$$
\text { Fluorescencia }(\mathrm{ml} / \mathrm{g})=\frac{F \times V}{S \times w}
$$

donde $\mathbf{V}(\mathrm{ml})$ es el volumen del extracto que produce la fluorescencia $\mathbf{F}$; w $(\mathrm{g})$ es el peso de la muestra empleada; $\mathbf{S}$ es la fluorescencia del estándar.

\subsection{Análisis estadístico}

Los datos obtenidos se sometieron a un análisis de varianza (ANOVA) de acuerdo con Sokal y Rohlf (1981). Se utilizó el nivel de significación $p<0,05$.

\section{RESULTADOS Y DISCUSIÓN}

\subsection{Humedad y contenido lipídico}

El contenido acuoso de la muestra refleja (Tabla I) valores típicos de sardinas y en general de pescado recién capturado (Piclet, 1987); se observó una ligera tendencia a aumentar con el tiempo de almacenamiento, aunque las diferencias únicamente son significativas si se comparan el principio y fin del estudio.

Tabla I

Humedad, contenido lipídico e índices lipídicos* de alteración para distintos tiempos de almacenamiento refrigerado de sardina**

\begin{tabular}{cccccc}
\hline $\begin{array}{c}\text { Tiempo } \\
\text { (días) }\end{array}$ & Humedad & $\mathrm{CL}$ & AGL & DC & i-TBA \\
\hline 0 & $69,02 \mathrm{a}$ & $3,69 \mathrm{ab}$ & $0,20 \mathrm{a}$ & $0,45 \mathrm{a}$ & $0,98 \mathrm{a}$ \\
& $(3,16)$ & $(0,59)$ & $(0,04)$ & $(0,06)$ & $(0,28)$ \\
2 & $70,64 \mathrm{ab}$ & $3,27 \mathrm{a}$ & $0,35 \mathrm{a}$ & $0,92 \mathrm{~b}$ & $2,07 \mathrm{ab}$ \\
& $(0,59)$ & $(0,77)$ & $(0,03)$ & $(0,11)$ & $(0,19)$ \\
6 & $72,56 \mathrm{~b}$ & $4,42 \mathrm{ab}$ & $0,35 \mathrm{a}$ & $1,11 \mathrm{~b}$ & $5,32 \mathrm{c}$ \\
& $(0,71)$ & $(0,79)$ & $(0,10)$ & $(0,18)$ & $(1,19)$ \\
9 & $71,25 \mathrm{ab}$ & $4,97 \mathrm{~b}$ & $1,14 \mathrm{~b}$ & $2,19 \mathrm{~d}$ & $6,02 \mathrm{c}$ \\
& $(1,14)$ & $(0,09)$ & $(0,27)$ & $(0,33)$ & $(0,46)$ \\
13 & $72,17 \mathrm{ab}$ & $4,55 \mathrm{ab}$ & $3,28 \mathrm{c}$ & $1,60 \mathrm{c}$ & $6,39 \mathrm{c}$ \\
& $(0,69)$ & $(0,54)$ & $(0,34)$ & $(0,17)$ & $(2,07)$ \\
16 & $73,79 \mathrm{~b}$ & $4,61 \mathrm{~b}$ & $2,88 \mathrm{c}$ & $0,98 \mathrm{~b}$ & $4,31 \mathrm{bc}$ \\
& $(0,76)$ & $(0,51)$ & $(0,19)$ & $(0,14)$ & $(0,35)$ \\
\hline
\end{tabular}

* Abreviaciones: CL (contenido lipídico), AGL (ácidos grasos libres), DC (dienos conjugados), i-TBA (índice de ácido tiobarbitúrico). Las unidades en que se representan se encuentran expuestas en la Parte Experimental.

** Valores medios de las determinaciones de los tres lotes del muestreo. En cada columna, los valores seguidos de letras distintas son significativamente distintos $(p<0,05)$. Se indica entre paréntesis el valor de la desviación estándar.
Los valores obtenidos para el contenido lipídico no mostraron prácticamente diferencias significativas (Tabla I). Se sabe que el contenido lipídico disminuye como resultado de someter el pescado a procesos que impliquen elevaciones de temperaturas como la cocción y la esterilización (Gallardo et al., 1989; Aubourg et al., 1995b); sin embargo en el caso de tratamiento por frío no se han descrito cambios. Los valores encontrados en el presente trabajo se ven influidos por el hecho de que únicamente se ha considerado el músculo blanco, despreciándose el rojo que tiene mayor contenido lipídico (Ackman, 1989).

\subsection{Hidrólisis y oxidación lipídicas}

La hidrólisis lipídica, medida a través del contenido en AGL refleja un incremento desde las muestras iniciales hasta los valores correspondientes a las dos últimas tomas (días 13 y 16, Tabla I). Los resultados demuestran cómo el índice de AGL es un buen método de seguimiento de la alteración de la muestra durante el almacenamiento refrigerado (Barassi et al., 1987), aunque en la medida en que la alteración está muy avanzada (13 y 16 días) su valor deja de aumentar debido a su descomposición en moléculas de menor peso molecular y consiguiente participación en las propiedades aromáticas del producto (Pearson et al., 1977).

La determinación de la oxidación lipídica se realizó en base a dos medidas: formación de dienos conjugados (oxidación primaria) y medición de compuestos reactivos con TBA (oxidación secundaria).

El contenido en dienos aumentó progresivamente hasta alcanzar un valor máximo el noveno día (Tabla 1); a partir de ese momento descendió de forma progresiva al aumentar el tiempo de almacenamiento. La cuantificación de dienos se ha empleado con buenos resultados en la medida de alteración incipiente en medios biológicos (Vossen et al., 1993); si embargo, en procesos que impliquen una alteración relativamente avanzada o temperaturas relativamente altas deja de ser válida como medida de alteración debido a ser moléculas inestables e interaccionar fácilmente con otros constituyentes (Cho et al., 1989; Aubourg et al., 1995a).

Se observó una tendencia ascendente en el índice de TBA, a medida que aumentaba el tiempo de almacenamiento (Tabla I). Sin embargo en la toma correspondiente al día 16 no se mantuvo dicho incremento. Nuevamente este ensayo deja de ser útil para seguir el estado de calidad en la medida en que se alcanza un estado de alteración relativamente elevado; este comportamiento se ha justificado como resultado de la facilidad de los compuestos carbonílicos para interaccionar con otros constituyentes de tipo amínico (Igene et al., 1979; Gardner, 1979; Kim y Labella, 1987). 


\subsection{Formación de fluorescencia}

Se estudió la fluorescencia de las distintas muestras a nivel de fases acuosa y orgánica (lipídica) resultantes de la extracción de lípidos según Bligh y Dyer (1959). De acuerdo con la bibliografía anterior (Aubourg et al., 1992a, 1992b y 1995a) se estudiaron cinco máximos de longitud de onda de excitación/emisión (327/415 nm, F1; 356/429 nm, F2; 393/463 nm, F3; 446/490 nm, F4; y 479/516 nm, F5). La medida a $528 / 556 \mathrm{~nm}$ reflejó resultados muy bajos, por lo que no se consideró oportuna la elaboración de sus resultados.

El estudio del extracto lipídico (Tabla II) indicó una tendencia del máximo $\mathrm{F} 1$ a disminuir a lo largo del almacenamiento, al tiempo que F2 se mantenía relativamente constante. Por su parte los máximos F3, F4 y F5 experimentaban aumentos en fluorescencia (mayoritariamente no significativos) a medida que aumentaba la alteración; de ellas, la medida F3 resultaba más sensible dado que F4 y F5 presentan valores relativamente más bajos.

Tabla II

Medida de fluorescencia en cinco máximos de excitación/emisión* sobre el extracto lipídico de sardina para distintos tiempos de refrigeración ${ }^{\star \star}$

\begin{tabular}{cccccc}
\hline $\begin{array}{c}\text { Tiempo } \\
\text { (días) }\end{array}$ & $\mathrm{F} 1$ & $\mathrm{~F} 2$ & $\mathrm{~F} 3$ & $\mathrm{~F} 4$ & $\mathrm{~F} 5$ \\
\hline 0 & $0,89 \mathrm{bc}$ & $0,57 \mathrm{ab}$ & $0,37 \mathrm{a}$ & $0,07 \mathrm{a}$ & $0,05 \mathrm{a}$ \\
& $(0,10)$ & $(0,05)$ & $(0,05)$ & $(0,00)$ & $(0,00)$ \\
2 & $0,97 \mathrm{c}$ & $0,69 \mathrm{bc}$ & $0,49 \mathrm{a}$ & $0,11 \mathrm{ab}$ & $0,07 \mathrm{ab}$ \\
& $(0,11)$ & $(0,13)$ & $(0,13)$ & $(0,04)$ & $(0,02)$ \\
6 & $1,04 \mathrm{c}$ & $0,60 \mathrm{abc}$ & $0,37 \mathrm{a}$ & $0,10 \mathrm{ab}$ & $0,07 \mathrm{ab}$ \\
& $(0,29)$ & $(0,10)$ & $(0,05)$ & $(0,03)$ & $(0,02)$ \\
9 & $0,64 \mathrm{ab}$ & $0,56 \mathrm{ab}$ & $0,46 \mathrm{a}$ & $0,20 \mathrm{~b}$ & $0,12 \mathrm{~b}$ \\
& $(0,10)$ & $(0,02)$ & $(0,05)$ & $(0,03)$ & $(0,02)$ \\
13 & $0,61 \mathrm{ab}$ & $0,52 \mathrm{a}$ & $0,39 \mathrm{a}$ & $0,14 \mathrm{ab}$ & $0,09 \mathrm{ab}$ \\
& $(0,10)$ & $(0,02)$ & $(0,02)$ & $(0,01)$ & $(0,01)$ \\
16 & $0,57 \mathrm{a}$ & $0,74 \mathrm{c}$ & $0,71 \mathrm{~b}$ & $0,32 \mathrm{c}$ & $0,20 \mathrm{c}$ \\
& $(0,06)$ & $(0,07)$ & $(0,15)$ & $(0,10)$ & $(0,08)$ \\
\hline
\end{tabular}

* Máximos de fluorescencia: F1 (327/415 nm), F2 (356/429 nm), F3 $(393 / 463 \mathrm{~nm}), \mathbf{F 4}(446 / 490 \mathrm{~nm})$ y F5 $(479 / 516 \mathrm{~nm})$.

** Valores medios de las determinaciones de los tres lotes del muestreo. En cada columna, los valores seguidos de letras distintas son significativamente distintos $(p<0,05)$. Se indica entre paréntesis el valor de la desviación estándar.

El estudio de la fase acuosa mostró (Tabla III) que el valor $\mathrm{F} 1$ tenía tendencia a disminuir aunque sin diferencias significativas, mientras que $\mathrm{F} 2$ aumentaba hasta la toma del décimotercer día, comenzando después a descender. Sin embargo los otros tres máximos (F3, F4 y F5) mostraron una tendencia a aumentar de forma continua a medida que crecía la alteración.
Tabla III

Medida de fluorescencia en cinco máximos de excitación/emisión* sobre el extracto acuoso de sardina para distintos tiempos de refrigeración ${ }^{\star \star}$

\begin{tabular}{cccccc}
\hline $\begin{array}{c}\text { Tiempo } \\
\text { (días) }\end{array}$ & $\mathrm{F} 1$ & $\mathrm{~F} 2$ & $\mathrm{~F} 3$ & $\mathrm{~F} 4$ & $\mathrm{~F} 5$ \\
\hline 0 & $4,59 \mathrm{a}$ & $0,69 \mathrm{a}$ & $0,35 \mathrm{a}$ & $0,26 \mathrm{a}$ & $0,46 \mathrm{a}$ \\
& $(0,14)$ & $(0,04)$ & $(0,03)$ & $(0,00)$ & $(0,02)$ \\
2 & $3,61 \mathrm{a}$ & $1,18 \mathrm{~b}$ & $0,33 \mathrm{a}$ & $0,36 \mathrm{a}$ & $0,69 \mathrm{ab}$ \\
& $(0,42)$ & $(0,10)$ & $(0,06)$ & $(0,02)$ & $(0,02)$ \\
6 & $4,26 \mathrm{a}$ & $2,16 \mathrm{~cd}$ & $0,43 \mathrm{a}$ & $0,52 \mathrm{~b}$ & $1,01 \mathrm{~b}$ \\
& $(2,56)$ & $(0,13)$ & $(0,08)$ & $(0,09)$ & $(0,16)$ \\
9 & $3,89 \mathrm{a}$ & $2,20 \mathrm{~cd}$ & $0,81 \mathrm{~b}$ & $0,62 \mathrm{~b}$ & $0,92 \mathrm{ab}$ \\
& $(0,74)$ & $(0,32)$ & $(0,15)$ & $(0,11)$ & $(0,11)$ \\
13 & $3,97 \mathrm{a}$ & $2,59 \mathrm{~d}$ & $0,80 \mathrm{~b}$ & $0,60 \mathrm{~b}$ & $1,18 \mathrm{bc}$ \\
& $(0,20)$ & $(0,14)$ & $(0,14)$ & $(0,09)$ & $(0,52)$ \\
16 & $2,61 \mathrm{a}$ & $1,88 \mathrm{c}$ & $1,05 \mathrm{c}$ & $0,95 \mathrm{c}$ & $1,67 \mathrm{c}$ \\
& $(0,31)$ & $(0,31)$ & $(0,08)$ & $(0,06)$ & $(0,08)$ \\
\hline
\end{tabular}

* Máximos de fluorescencia: F1 (327/415 nm), F2 (356/429 nm), F3 $(393 / 463 \mathrm{~nm}), \mathbf{F 4}(446 / 490 \mathrm{~nm})$ y F5 $(479 / 516 \mathrm{~nm})$.

** Valores medios de las determinaciones de los tres lotes del muestreo. En cada columna, los valores seguidos de letras distintas son significativamente distintos $(p<0,05)$. Se indica entre paréntesis el valor de la desviación estándar.

En comparación con los extractos lipídicos, la fase acuosa reflejó medidas mayores y más sensibles, dando a entender que una parte mayoritaria de los compuestos de interacción portadores de propiedades fluorescentes son más solubles en el medio acuoso que en el orgánico.

Los estudios de correlación de formación de compuestos fluorescentes con calidad de muestras de alimentos se han venido centrando en los extractos orgánicos o lipídicos, llegándose a obtener una buena relación con las medidas sensoriales y tiempo de almacenamiento (Maruf et al., 1990; Lubis y Buckle, 1990). Sin embargo otras investigaciones han llegado a proponer que los compuestos responsables de la fluorescencia son fundamentalmente compuestos derivados de las proteínas y por tanto poco solubles en la fase orgánica (lio y Yoden, 1988; Hasegawa et al., 1992).

Los resultados obtenidos en el presente trabajo apoyan la importancia de la fase acuosa como sustrato de medición de las propiedades fluorescentes. El desplazamiento de la fluorescencia a mayores longitudes de onda estaría de acuerdo con la teoría general referente a la formación progresiva de compuestos de interacción con mayor peso molecular y grado de insaturación a medida que aumenta la alteración (Pokorný, 1977; Gardner, 1979); además se ve facilitada por la capacidad catalítica de los compuestos amínicos sobre la condensación aldólica de los productos de oxidación secundaria lipídica que se forman como resultado de la alteración (Suyama et al., 1981 Pokorný et al., 1987). 


\subsection{Comparación del desplazamiento de fluorescencia con los índices lipídicos tradicionales}

En los resultados obtenidos en el presente trabajo se ha podido observar cómo la medida del índice de TBA y de AGL, y sobre todo la de dienos conjugados, no eran sensibles a la hora de seguir el grado de alteración cuando la muestra estaba en un estado de degradación avanzada (Tabla I).

Por su parte, la medida de ciertos máximos de fluorescencia (F3, F4 y F5) mostró un incremento más o menos continuo a medida que aumentaba el tiempo de almacenamiento refrigerado, y con él, la alteración de los lípidos de la muestra. En trabajos anteriores referentes a tratamientos térmicos (Aubourg et al., $1992 b$ y 1995a) se consideró como interesante el estudio de la relación entre la fluorescencia de los máximos F3 y F1; dicha relación se observó que se correlacionaba bien con la intensidad del tratamiento térmico.

En el presente experimento se estudió este desplazamiento de fluorescencia (F3/F1) para los extractos acuoso y orgánico (Figura 1). Ambas fases mostraron una tendencia a aumentar a medida que se prolongaba el tiempo de almacenamiento, contraponiéndose a los índices lipídicos. Sin embargo se puede observar cómo en el caso de la fase acuosa se produce un incremento más continuo y proporcionado a lo largo de todo el almacenamiento. Esta diferencia se puede comprobar al estudiar la correlación entre el tiempo de refrigeración y el desplazamiento de fluorescencia. La correlación fue mejor en el caso de la fase aucosa $(r=0,8533)$ que en el de la orgánica $(r=0,7457)$.

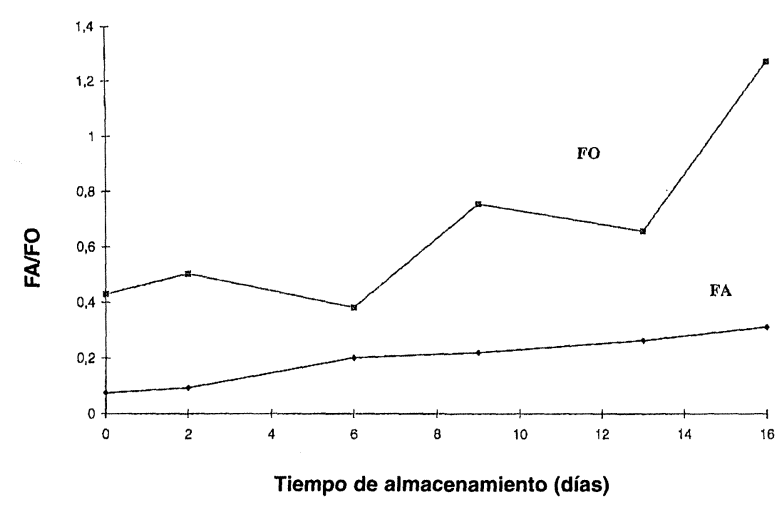

Figura 1

Evolución del desplazamiento de fluorescencia $(F 3 / F 1)^{\star}$ en las fases acuosa (FA) y orgánica (FO) durante el almacenamiento refrigerado de sardina

* Calculado como la relación entre la fluorescencia a 393/463 $\mathrm{nm}(\mathrm{F} 3)$ y a $327 / 415 \mathrm{~nm}(\mathrm{~F} 1)$. Valores medios de las determinaciones de los tres lotes del muestreo.
Como resultado del presente estudio, se concluye que el análisis de los compuestos de interacción a través de sus propiedades fluorescentes resulta útil al objeto de correlacionarlo con la calidad de una muestra de pescado que ha sido almacenada en estado refrigerado. Es asimismo de resaltar la mayor sencillez y rapidez que este método fluorimétrico supone frente a los métodos químico tradicionales.

\section{AGRADECIMIENTOS}

Los autores agradecen a don Marcos Trigo y don Oscar Bellón su eficaz colaboración en el desarrollo del presente trabajo, y a la Xunta de Galicia la ayuda financiera otorgada (Proyecto XUGA 402 01B 93).

\section{BIBLIOGRAFÍA}

Ackman, R. (1989). - «Fatty acids». En «Marine biogenic lipids, fats and oils".- R. Ackman (editor).- CRC Press, Boca Raton, FI. Vol. 1, pp. 103-137.

Aubourg, S., Pérez-Martín, R., Medina, I. y Gallardo, J. (1992a). - «Fluorescence formation by interaction of albacore (Thunnus alalunga) muscle with acetaldehyde in a model system".- J. Agric. Food Chem. 40, 1805-1808.

Aubourg, S., Pérez-Martín, R., Medina, I. y Gallardo, J. (1992b). - «Fluorescence formation during albacore (Thunnus alalunga) thermal processing".- Z. Lebensm. Unters. Forsch. 195, 332-335.

Aubourg, S., Medina, I. y Pérez-Martín, R. (1995a). -«A comparison between conventional and fluorescence detection methods of cooking-induced damage to tuna fish lipids". - Z. Lebensm. Unters. Forsch. 200, 252-255.

Aubourg, S., Medina, I., Gallardo, J. y Pérez-Martín, R. (1995b). - «Efecto del enlatado en aceite y salmuera y su posterior almacenamiento sobre los lípidos de la bacoreta (Euthynnus alletteratus)".- Grasas y Aceites 46, 77-84.

Aursand, M., Bleivik, B., Reinuzzo, J., Jörgensen, L. y Mohr, V. (1994). - «Lipid distribution and composition of commercially farmed atlantic salmon (Salmo salar)".- J. Sci. Food Agric. 64, 239-248.

Barassi, C., Pécora, R., Roldán, H. y Trucco, R. (1987). - «otal, non-volatile free fatty acids as a freshness index for hake (Merluccius hubbsi) stored in ice».-J. Sci. Food Agric. 38, 373-377.

Bligh, E. y Dyer, W. (1959). - «A rapid method of total extraction and purification".-Can. J. Biochem. Physiol. 37, 911-917.

Cheftel, J. y Cheftel, H. (1976). - «Introducción a la Biología y Tecnología de alimentos".- Editorial Acribia, Zaragoza.

Cho, S. Y., Endo, Y., Fujimoto, K. y Kaneda, T. (1989). - «Autoxidation of ethyl eicosapentaenoate in a defatted fish dry model system".- Nippon Suisan Gakkaishi 55, 545-552.

Gallardo, J., Aubourg, S. y Pérez-Martín, R. (1989). -«Lipid classes and their fatty acids at different loci of albacore (Thunnus alalunga): Effect of precooking".- J. Agric. Food Chem. 37, 1060-1064.

Gardner, H. (1979). - «Lipid hydroperoxide reactivity with proteins and amino acids: A review".- J. Agric. Food Chem. 27, 220-229. 
Hasegawa, K., Endo, Y. y Fujimoto, K. (1992). - «Oxidative deterioration in dried fish model systems assessed by solid sample fluorescence spectrophotometry".- J. Food Sci. 57, 1123-1126.

Herbes, S. y Allen, C. (1983). - «Lipid quantification of freshwater invertebrates: method modification for microquantification».Can. J. Fish. Aquat. Sci. 40, 1315-1317.

Hidalgo, F., Zamora, R., Aláiz, M., Maza, M., Millán, F. y Vioque, E. (1990). - «Nonenzymatic browning reactions of phospholipids".- Fat Sci. Technol. 92, 185-188.

Igene, J., Pearson, A., Merkel, R. y Coleman, T. (1979). - «Effect of frozen storage time, cooking and holding temperature upon extractable lipids and TBA value of beef and chicken».- J. Anim. Sci. 49, 701-707.

lio, T. y Yoden, K. (1988). - «Fluorescence formation from hydroperoxide of phosphatidylcholine with amino compound".- Lipids 23, 65-67.

King, R. y Labella, F. (1987). - "Comparison of analytical methods for monitoring autoxidation profiles of authentic lipids».- J. Lipid Res. 28, 1110-1117.

Lowry, R. y Tinsley, I. (1976). - «Rapid colorimetric determination of free fatty acids".- J. Amer. Oil Chem. Soc. 53, 470-472.

Lubis, Z. y Buckle, K. (1990). -«Rancidity and lipid oxidation of dried-salted sardines».- Int. J. Food Sci. Technol. 25, 295-303.

Maruf, F., Ledward, D., Neale, R. y Poulter, R. (1990). - "Chemical and nutritional quality of Indonesian driedsalted mackerel (Rastrelliger kanagurta)».- Int. J. Food Sci. Technol. 25, 66-77.

Melton, S. (1983). - «Methodology for following lipid oxidation in muscle foods".- Food Technology 37, 105-111, 116.

Pearson, A., Love, J. y Shorland F. (1977). -«Warmed-over flavor in meat, poultry and fish".- Adv. Food Res. 23, 2-61.

Piclet, G. (1987). - «Le poisson aliment. Composition, intérêt nutritionnel».- Cah. Nutr. Diét. XXII, 317-335.

Pokorny, J. (1977). - - Interactions of oxidized lipids with proteins».- Riv. Ital. Sostanze Grasse 54, 389-393.

Pokorny, J., Janitz, W., Víden, I., Velísek, J., Valentová, H. y Davídek, J. (1987). - «Reaction of oxidized lipids with protein. Part 14. Aldolization reactions of lower alkanals in presence of nonlipidic substances".- Nahrung 31, 63-70.

Smith, G., Hole, M. y Hanson, S. (1990). - «Assessment of lipid oxidation in Indonesian salted-dried Marine catfish (Arius thalassinus)".- J. Sci. Food Agric. 51, 193-205.

Sokal, R. y Rohlf, F. (1981). -«Biometry».- Segunda edición. Editorial W. Freeman and Company, San Francisco.

Suyama, K., Arakawa, T. y Adachi, S. (1981). - «Free fatty aldehydes and their aldol condensation products in heated meat».- J. Agric. Food Chem. 29, 875-878.

Vossen, R., van Dam-Mieras, M., Hornstra, G. y Zwaal, R. (1993). - «Continuous monitoring of lipid peroxidation by measuring conjugated diene formation in an aqueous liposome formation".- Lipids 28, 857-861.

Vyncke, W. (1970). - -Direct determination of the thiobarbituric acid value in trichloracetic acid extracts of fish as a measure of oxidative rancidity".- Fette, Seifen, Anstrichm. 72, 1084-1087.

Zamora, R., Millán, F., Hidalgo, F., Aláiz, M., Maza, M. y Vioque, E. (1986). - «Aplicaciones de la fluorescencia al estudio de las interacciones lípido-proteína. Ensayos previos».- Grasas y Aceites 37, 317-319. 